\title{
Biofilm growth and hydrodynamic behaviour in the biological plate tower (BPT) with and without hanging biomass (BPT-HB)
}

\author{
João Monteiro Peixoto and João Carlos Ribeiro Pinto
}

\begin{abstract}
Deodorization and volatile organic compound abatement from polluted air streams can be accomplished with the biological plate tower (BPT), which has proved to be a reliable alternative to biofilters and biotrickling filters. Unlike those, the BPT is a non-clogging device, with constant active surface, and steady performance, making it ideal for scale-up and modelling. The initial BPT design needed improvement for better performance. The cylindrical body (circular plates) was replaced by a rectangular cuboid (rectangular plates). Holes in the plates did augment the amount of active biomass (hanging from the holes and under the plates), without reducing the transfer of toluene from the gaseous to the liquid phase. The diminished distance between plates was well tolerated in cocurrent flow, allowing much higher quantities of biomass in the same reactor volume. With 18 and $14 \mathrm{~mm}$ spacing between adjacent plates, the BPT, with and without holes, was tested for flooding, holdup and pressure drop. Several gas and liquid flows were tested, both in co-current and countercurrent. In hydrodynamic terms, the BPT-HB with co-current flow was clearly the best option. Higher stability with higher flow rates, and lower pressure drops were observed. The inoculum was obtained from wastewater plant activated sludge (petrochemical industry).
\end{abstract}

Key words | biofiltration, BPT-HB, co-current, hydrodynamic, odour, VOC

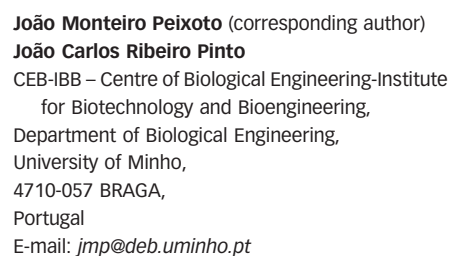

\section{INTRODUCTION}

Scientists have the responsibility to find the most efficient and economical processes. Odour and volatile organic compound (VOC) air pollution have reached objectionable proportions. Undesirable odours contribute to air quality concerns and affect human lifestyles. Odour-causing substances have health effects such as eye, nose and throat irritation, headache, and drowsiness, and possibly aggravate allergies, asthma, and bronchitis (Sohn et al. 2003; Bard et al. 20Io). More frequently, strong, unpleasant, or offensive smells can interfere with a person's enjoyment of life, even if malodours are inoffensive (citizens' protest). The relevant odours are nitrogen-containing compounds (e.g., ammonia, amines, amides, indole and scatole), reduced sulphur compounds and VOCs like alcohols, aldehydes, volatile fatty acids, and phenols. Most of the work has focused on ammonia nitrification, as well as the treatment of reduced sulphur compounds (single or mixture) and odourous VOCs.
Most VOCs are photochemical substances related to high atmospheric levels of ozone (acute respiratory disease, material corrosion and ecosystem damages) in highly polluted urban environments during the summer peaks of solar activity. Some are toxic, and some are carcinogenic (e.g., benzene) and even mutagenic. Some VOCs are also bad odours. All these compounds present global warming potentials much higher than that of $\mathrm{CO}_{2}$ 's. Their oxidation creates $\mathrm{CO}_{2}$ and biomass (that may be used to produce methane and energy and will positively contribute to the global warming solution). Targets are the wood and paint industries, as well as odours related to wastewater treatment plants and sanitary landfills.

Legislation is more restrictive about odour and VOC emissions and ozone control. Source control eliminates the pollutants or reduces their emissions, and is generally the most effective strategy. Large air-flow rates with low pollutant concentrations frequently are encountered. Deodorization and VOC abatement can be accomplished 
by methods such as condensation, adsorption (e.g., activated carbon and zeolites), absorption (wet scrubbing) and membrane technologies. Destructive methods promote the oxidation of pollutants as in thermal oxidizers (direct or catalytic) and biological filtration (Rafson 1998). Biological methods are non-hazardous and benign. Usually no secondary wastes are produced. They are cost effective, when low concentrations ( 1 to $10 \mathrm{mg} / \mathrm{L}$ ) are to be dealt with (Kosteltz et al. 1996). Most odours and VOCs are easily biodegradable.

Several biological reactor concepts exist. The filter material (organic or inorganic) and the liquid phase (continuous or not) distinguish them. Pollutants are first transferred from the gas to the liquid phase and subsequently to the biofilm. Laboratory studies provide much information about the removal of single compounds or simple mixtures. Pilot-scale experiments are conducted onsite to investigate performance and design criteria, as recommended by Devinny et al. (I999). Bioreactors may be classified into five groups: biofilters $(\mathrm{BF})$, biotrickling filters (BTF), biowashers (BW) (e.g., bioscrubbers), the biological plate tower (BPT) and the membrane bioreactor (MB). Deodorization and VOC abatement from polluted air streams can be accomplished with the BPT, which has proved to be a reliable alternative to $\mathrm{BF}$ and BTF. Unlike those, which are mostly applied, the BPT is a non-clogging device, with constant active surface, and steady performance, making it ideal for scale-up and modelling.

Biofilms play an important role in protecting the microorganisms (toxic or aggressive conditions). Attached biomass is less prone to inhibition, destruction or washout. $\mathrm{BF}, \mathrm{BTF}, \mathrm{BPT}$ and airlift reactors (as a part of a BW), all grow attached biofilms. The absence of a moving liquid phase is the big disadvantage of BF. For the control of temperature, $\mathrm{pH}, \mathrm{O}_{2}$, and nutrient supply, the presence of liquid water is a major advantage. Biofilm growth is chaotic on a random packing. Four-phase (liquid, gas, support and biofilm) reactors always bring about hydrodynamic problems. Channelling, clogging and sloughing are three occurrences when classical biological reactors are used. The most widely applied reactors are BF. To overcome channelling and clogging they are usually over-dimensioned but will have variable performances over time. They have decays that are difficult to predict and model. Therefore, they are not really scientific devices. Their performance is never stable. The very important properties of the reactor media (Shareefdeen et al. 2005) may not be that important, except for the ability to support bacterial adhesion.

Several packing materials were checked to try to avoid channelling and clogging (Peixoto \& Mota 1998), without success. As a solution to those problems, the research evolved to the BPT. It has been proved so far that it solves the cited problems and has a very good performance. Mass transfer, air to liquid, is also very good due to the existence of both parallel and cross flow (Sercu et al. 2006; Mota \& Peixoto 2008). Clogging is a direct result of the bacterial (fungal) growth, due to pollutant degradation. It does not make sense to try to limit the growth to avoid it. The BPT is the solution. The BPT resulted in high odour and VOC removal (above 90\% removal for inlet ammonium concentrations between 7.3 and $136.6 \mu \mathrm{g} / \mathrm{L}$ and up to $25 \mathrm{mg} / \mathrm{L}$ for toluene).

The present work aimed to enhance the BPT performance by changing its geometry from cylindrical (circular plates) to a rectangular cuboid (rectangular plates) and testing the hydrodynamic behaviour of co-current versus counter-current flows (flooding, holdup and pressure drop) with diminished distance between adjacent plates. Indeed, in recent experiments, some hydrodynamic difficulties related to high air flows and/or biofilm growth suggested that co-current flow should be tried. With aromatic VOCs the thick biofilms obtained suggested that the presence of holes in the plates might augment the amount of active biomass (hanging from the holes and under the plates).

\section{METHODS}

The first design of the BPT was a pile of parallel circular plates with a single hole on the border (Sercu et al. 2006), alternating $\left(180^{\circ}\right)$ from one to the next plate. A cascade of liquid went down, changing direction from plate to plate. The gaseous stream followed the opposite direction, upward. The bacteria attached to the top surface of the plates. For this research, a new BPT was built. A rectangular cuboid (rectangular plates) replaced the first version's cylindrical shape. The volume was reduced allowing higher security level (lower flows/pressures, lower pollutant volumes and concentrations) for laboratory research. There were two $8.6 \mathrm{dm}^{3}$ modules, containing two separate sets of plates (two submodules), allowing for three gas sampling points (in, middle and out) in each module. The rectangular plates ensure more even distribution of both liquid and gaseous flows. Each plate measured $128 \times 168 \times 4 \mathrm{~mm}^{3}$, leaving $20 \times 128 \mathrm{~mm}^{2}$ free to guarantee the fluid circulation. One module used pierced plates (plates with holes) as presented in Figure 1.

With $18 \mathrm{~mm}$ (nine plates per submodule) and $14 \mathrm{~mm}(11$ plates per submodule) spacing between adjacent plates, the 


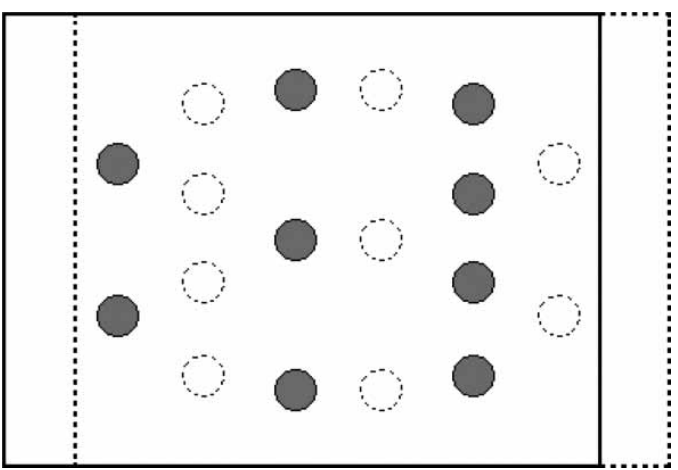

Figure 1 | Relative position of the $12 \mathrm{~mm}$ diameter holes in two adjacent plates (top view).

BPT, with pierced and non-pierced plates, was tested for flooding, holdup, pressure drop and void fraction. Several gas and liquid flow combinations were tested, both in co-current and counter-current.

The laboratory installation included a $6.8 \mathrm{dm}^{3}$ fermentor, Setric Genie Industrial (SGI), for inocula development and $\mathrm{pH}$ and temperature control. A couple of pumps, Grundfos-UP 20-45 N 150, were used to move the liquid phase, controlled by two rotameters, Fisher \& Porter, D 10 A 1197 A, D 055. The effluent simulation was achieved with the special mixing chamber described in Peixoto \& Mota (I997), but modified by the inclusion of a conic body to regulate the evaporating area available. The compressed air system supplied the air and a Gilmont flowmeter, calibrated in operational conditions, allowed control of the flow rate.

The pressure drop measurement device was a water $\mathrm{U}$ glass manometer. Although liquid holdup was also determined, the pressure drop is of uttermost importance for the follow-up of the operation. Pressure drop is the best parameter for deciding whether to change a set of plates and replace it with a new one that should be previously inoculated to perform adequately from the start. In BFs the pressure drop is also a very important control parameter (Öscan 2006).

Liquid holdup informs about the average water depth circulating on each plate and dropping in the holes (if present) and the cascades. Higher volumes will be responsible for higher pressure drops. The best holdup guarantees the ideal turbulence for mass transfer without causing excessive pressure drop. To measure it, the circulating liquid input and output are simultaneously cut and the gain of volume above the stationary level is calculated.

The inoculum was selected according to the conclusions of Mota \& Peixoto (2008). An industrial petrochemical wastewater plant, contaminated with aromatic compounds, was the source of microorganisms. The carbon source must never be an easily degradable substrate (e.g., glucose) but those they are meant to degrade. Toluene was used in this research. The biofilm growth causes a progressive reduction of the void fraction. By flooding the reactor and measuring the volume of liquid needed it is possible to determine the void fraction and make a good estimate of the biofilm thickness. With the pierced plates this procedure is, obviously, more complicated.

\section{RESULTS AND DISCUSSION}

The holdup volumes, $H V$, before the biofilm growth, with pierced and non-pierced plates, for co-current and countercurrent flows, can be observed in Figure 2. There is a clearly
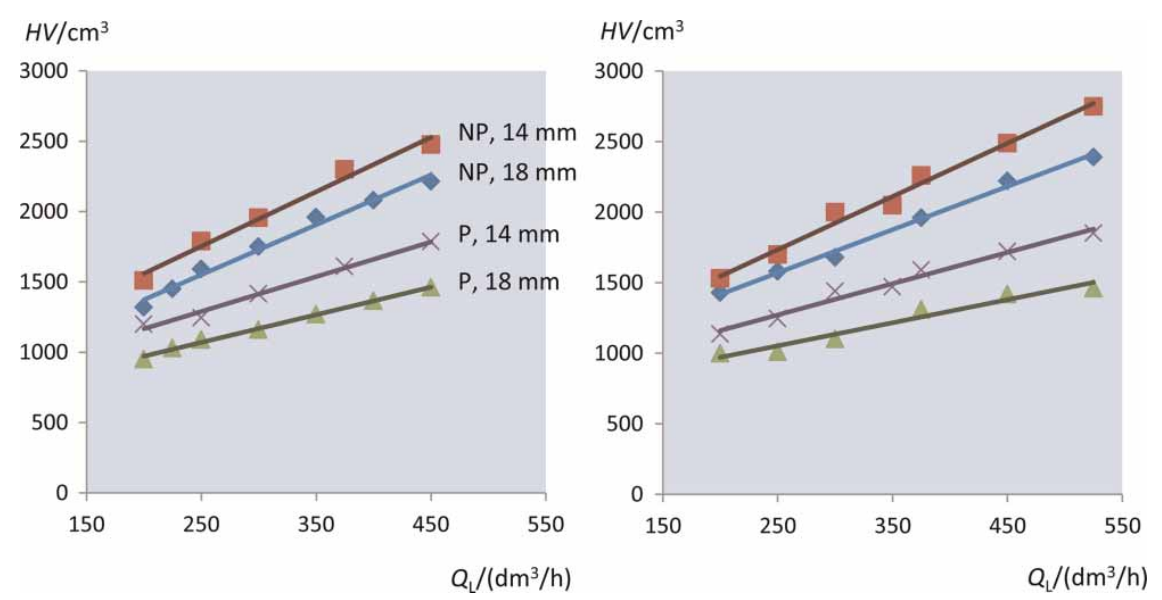

Figure 2 | Holdup volumes, $H V$, as a function of the liquid flow rates, $Q_{\mathrm{L}}$, for counter-current (left) and co-current (right) operation. NP and P stand for non-pierced and pierced plates. 
linear relation between $H V$ and liquid flow rate, $Q_{\mathrm{L}}$. The gas flow rate, $Q_{\mathrm{G}}$, didn't significantly change the $H V$ values. For the same distance between equal plates, the effect of changing from co-current to counter-current was very discrete and the corresponding straight lines present very similar slopes. During the biofilm growth, $Q_{\mathrm{L}}$ was set to $200 \mathrm{dm}^{3} /$ h. In the end (after $50 \mathrm{~d}$ ), $H V=1.18 \mathrm{dm}^{3}$ in the module with pierced plates (it was equal to $1.20 \mathrm{dm}^{3}$ before the colonization) and $H V=1.60 \mathrm{dm}^{3}$ in the other one $\left(1.51 \mathrm{dm}^{3}\right.$ before colonization). It is clear that $H V$ is much lower in the module with pierced plates, with both modes of operation. The space between plates causes just a little variation in the $H V$ value.

As expected, pressure drops in the gaseous stream $(\Delta p)$ were higher for higher flow rates. Tested values of $Q_{\mathrm{L}}$ ranged from 200 to $525 \mathrm{dm}^{3} / \mathrm{h}$. For $Q_{\mathrm{G}}$ the range went from 45 to $813 \mathrm{~cm}^{3} / \mathrm{s}$. With the lower $Q_{\mathrm{L}}$ (in both modes of operation and both distances between plates), $\Delta p$ never exceeded $0.36 \mathrm{kPa}$ (Figure 3), in the range of values presented by Wang et al. (2009) for BFs. For higher values of $Q_{\mathrm{L}}$ and $Q_{\mathrm{G}}, \Delta p$ was still kept below that value in co-current flow. Only with high values of $Q_{\mathrm{L}}$ and $Q_{\mathrm{G}}$, in countercurrent flow, the problems did appear, as can be observed in Figure 3. In fact, with $Q_{\mathrm{L}}=450 \mathrm{dm}^{3} / \mathrm{h}$ and $Q_{\mathrm{G}}=449 \mathrm{~cm}^{3} / \mathrm{s}$, a situation of pre-flooding arose in the upper submodule with non-pierced plates. When $Q_{\mathrm{G}}$ reached $763 \mathrm{~cm}^{3} / \mathrm{s}$, flooding occurred. With pierced plates the problem was not as severe.

Adding the biomass to the plates, all the hydrodynamic problems became more evident and, in the end, $\Delta p$ was more difficult to measure due to the presence of higher liquid volumes that invaded the connections to the manometer, and only the lower values of $Q_{\mathrm{L}}$ and $Q_{\mathrm{G}}$ might be implemented in counter-current mode.

Before inoculation and not considering the holdup volumes, the void fraction was 0.882 and 0.876 , respectively for pierced and non-pierced $18 \mathrm{~mm}$ spacing modules. The corresponding values for $14 \mathrm{~mm}$ spacing were 0.856 and 0.849 . Due to the necessary long time, only one assay of biomass growth took place, using the modules with $14 \mathrm{~mm}$ plate separation. The biofilm developed normally in both modules, as can be observed in Figures 4 and 5 . The biomass caused the void fraction to drop to 0.762 and 0.721 for the pierced and non-pierced plates, respectively. The difference represents the volume occupied by the biofilm. The abundant biofilm confirms, as in previous works, that mass transfer (oxygen and toluene) between the two streams was very efficient.

In both modules, the biofilm thickness was bigger in the lower submodules because the development of the biofilm was done in counter-current flow. Therefore, the toluene concentration was higher in the inlet region. Anyway, the huge biofilms obtained in previous research suggested that the biofilms hanging from the holes (Figure 6) would be big enough to connect the plates and also colonize the underside of the face. Time was not long enough to reach that situation. If so, the active surface would be really very interesting. Removal in the modules with pierced plates might be three times higher than in the non-pierced ones. Nevertheless, in Figure 6 the hanging biomass is perfectly visible. Still, there is a very poor colonization of the underside face of the plate. The explanation is, probably, the difference between the inocula. The biggest hanging formations were obtained with a pure strand
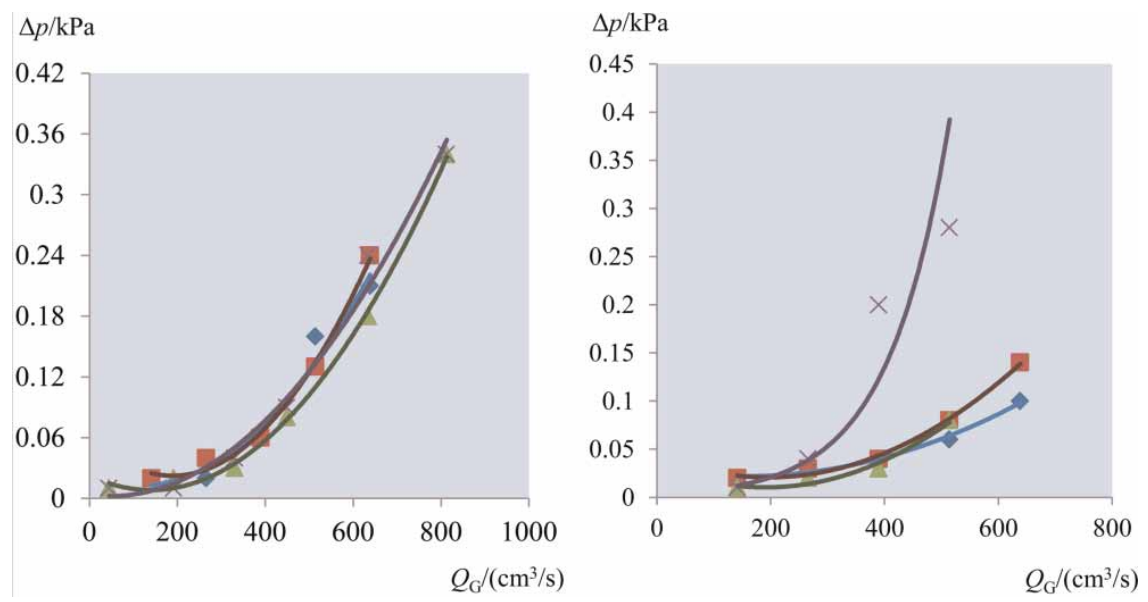

Figure 3 Similar pressure drops for all combinations of $18 \mathrm{~mm}$ spacing and both modes of operation, with $Q_{\mathrm{L}}=200 \mathrm{dm}^{3} / \mathrm{h}$ (left). For $Q_{\mathrm{L}}=450 \mathrm{dm} / \mathrm{h}$ (right) and $14 \mathrm{~mm} \mathrm{spacing,} \mathrm{only} \mathrm{the}$ combination of counter-current mode with non-pierced plates presents an early problem. 

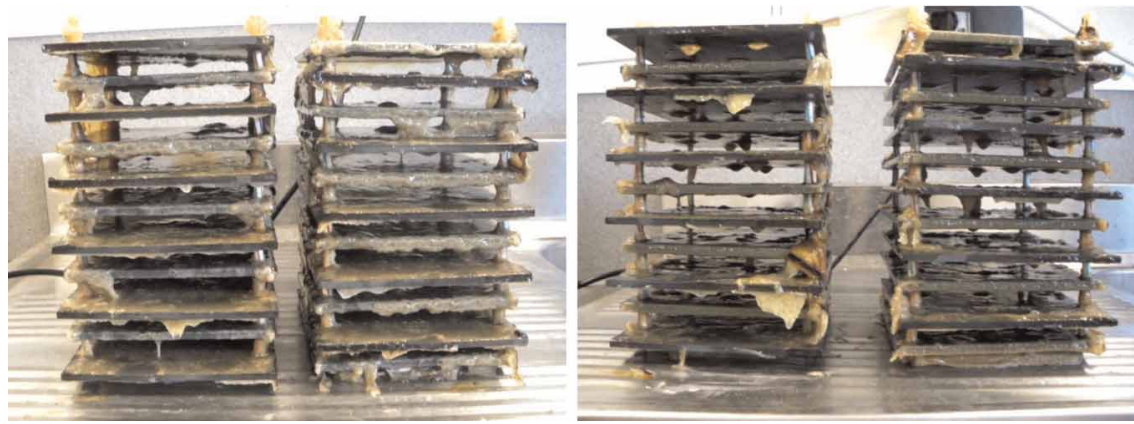

Figure 4 | Photographs of the two submodules with non-pierced plates (left) and with pierced plates (right), showing the grown biofilms on the plates and hanging from the cascades and from the holes.

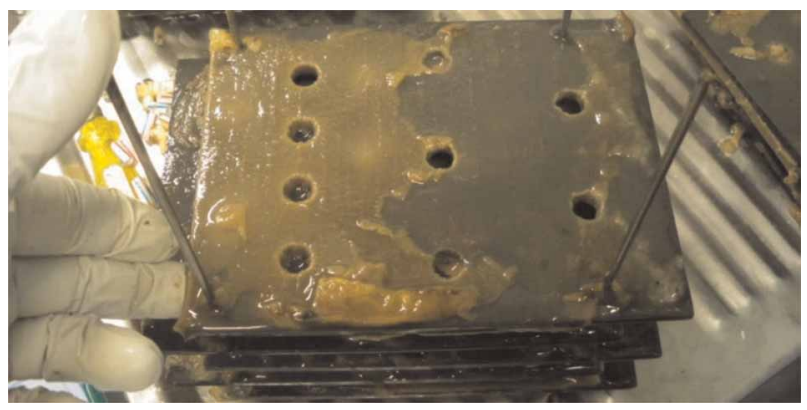

Figure 5 | Photograph with the partially removed biofilm on the top of a pierced plate.

of bacteria, Pseudomonas putida ATCC 17514, that also possessed very good characteristics for the colonization of the underside faces of the plates. Pure strands have the advantage of being bought ready to start without the need for acclimatization. On the other hand, they sometimes lose their inductive enzymes and acclimatization must be undertaken. One way or the other, bacteria must always be kept under stress.

\section{CONCLUSIONS}

The holes in the plates did augment the amount of active biomass (hanging from the holes and under the plates), without reducing the transfer of toluene from the gaseous to the liquid phase. The diminished distance between plates was well tolerated in co-current flow (no flooding occurrence was observed), allowing much higher quantity of biomass in the same reactor volume, with a clear possibility to enhance the reactor performance. The transparent front wall of the reactor (opaque in the previous versions) proved to be a great idea, because it was possible to observe the flooding condition and appropriately adjust the values of $Q_{\mathrm{L}}$ and $Q_{\mathrm{G}}$.

For future research, the plate sets will have a bigger number of plates (shorter distances between plates) and the number of holes should be increased. For nitrification processes, the very thin biofilms obtained will allow a minimal distance between plates but the presence of any holes in the plates has to be better studied. Co-current flow is definitely the best option in all situations.

The hanging biofilms did not form evenly and were much shorter and more detachable and fragile when compared with the ones obtained with P. putida ATCC 17514. Anyway, they might be more interesting if a longer period had been given to the assay. Complex inocula are to be preferred for the simulation of natural conditions but pure strands are still to be considered during inocula selection.
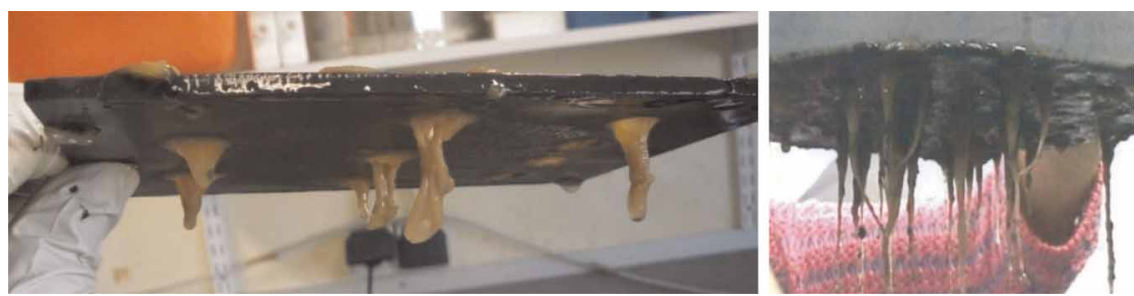

Figure 6 | Photographs showing hanging biomass: from a pierced plate of the present research (left) and from the liquid distributor of previous works using a pure strand of $P$. putida. Note the dimension of each formation comparing with the thumb (left) and the arm (right). 


\section{ACKNOWLEDGEMENTS}

The authors acknowledge the important contribution of Master João Jorge Vilela, during the construction and firsttime operation of the bioreactor.

\section{REFERENCES}

Bard, D., Laurent, O., Havard, S., Deguen, S., Pedrono, G., Filleul, L., Segala, C., Lefranc, A., Schillinger, C. \& Riviere, E. 2010 Ambient air pollution, social inequalities and asthma exacerbation in Greater Strasbourg (France) Metropolitan area: the PAISA study. In: Air Pollution (V. Villanyi ed.). Sciyo, Rijeka, Croatia.

Devinny, J. S., Deshusses, M. A. \& Webster, T. S. 1999 Biofiltration for Air Pollution Control. Lewis Publishers, Boca Raton, FL, USA.

Kosteltz, A. M., Finkelstein, A. \& Sears, G. 1996 What are the 'real opportunities' in biological gas cleaning for North America? In: Proceedings of the 89th Annual Meeting \& Exhibition of A\&WMA, Air and Waste Management Association, Pittsburgh, PA; 96-RA87B.02.

Mota, M. \& Peixoto, J. 2008 Inocula selection for VOC removal in the non-clogging biological plate tower. In: Book of Abstracts of the 10th International Chemical and Biological Engineering Conference - CHEMPOR 2008 (E. C. Ferreira \&
M. Mota, eds). University of Minho, Braga, Portugal, September 4-6, 2008, pp. 933-934.

Öscan, E. B. 2006 Agricultural Waste Products as Filter Media and as Cover Materials in Biofilters for Mediterranean Countries. Oldenbourg Industrieverlag, $\mathrm{GmbH}$, Munich.

Peixoto, J. \& Mota, M. 1997 Volatile organic compounds evaporation chamber for the simulation of gas effluents in laboratory research. Biotechnology Techniques 11, 1-6.

Peixoto, J. \& Mota, M. 1998 Biodegradation of toluene in a trickling filter. Bioprocess Engineering 19 (5), 393-397.

Rafson (ed.) I998 Odor and Voc Control Handbook. McGraw-Hill, New York

Sercu, B., Peixoto, J., Demeestere, K., van Helst, T. \& van Langenhove, H. 2006 Odors treatment: biological technologies. In: Odors in the Food Industry (Xavier Nicolay, ed.). Springer, pp. 125-158.

Shareefdeen, Z., Herner, B. \& Singh, A. 2005 Biotechnology for air pollution control - an overview. In: Biotechnology for Odor and Air Pollution Control (Z. Shareefdeen \& A. Singh, eds). Springer-Verlag, Berlin.

Sohn, J. H., Smith, R., Yoong, E., Leis, J. \& Galvin, G. 2003 Quantification of odours from piggery effluent ponds using an electronic nose and an artificial neural network. Biosystems Engineering 86 (4), 399-410.

Wang, L. K., Shammas, N. K., Guild, J. \& Pollock, D. 2009 Vertical shaft digestion, flotation and biofiltration. In: Advanced Biological Treatment Processes (L. K. Wang, N. K. Shammas \& Y. Hung, eds). Humana Press, New York. 